\title{
Human Recombinant Granulocyte-Macrophage Colony Stimulating Factor and Interleukin 3 Have Overlapping but Distinct Hematopoietic Activities
}

\author{
Stephen G. Emerson, ${ }^{\star}$ Yu-Chung Yang, ${ }^{\ddagger}$ Steven C. Clark, ${ }^{\ddagger}$ and Michael W. Long ${ }^{\$}$ \\ Departments of *Internal Medicine and ${ }^{\S}$ Pediatrics, University of Michigan Medical Center, Ann Arbor, Michigan 48109; \\ and ${ }^{\ddagger}$ Genetics Institute, Cambridge, Massachusetts 02140
}

\begin{abstract}
The hematopoietic stimulatory activities of human recombinant IL-3 and granulocyte-macrophage colony stimulating factor (GM-CSF) were directly compared using highly enriched human bone marrow progenitor target cells. IL-3 supported a larger number of erythroid and megakaryocytic progenitor cells than did GM-CSF, while GM-CSF supported more myeloid progenitors. IL-3 directly stimulated the division and migration of primitive erythroid burst forming units, while GMCSF merely sustained their net survival in culture without promoting division and expansion. IL-3 promoted the formation of larger numbers of multipotential granulocyte-erythroid-macrophage-megakaryocyte colony forming unit-derived colonies than did GM-CSF. These data indicate that human IL-3 and GM-CSF have overlapping but distinct hematopoietic activities, and suggest a potential role for the clinical application of combined IL-3/GM-CSF therapy.
\end{abstract}

\section{Introduction}

Hematopoietic differentiation is a complex process in which self-renewing pluripotent stem cells divide and evolve into committed progenitor cells, recognizable precursor cells, and finally mature blood cells. Although the factors that regulate the earliest stages of stem cell differentiation are not well described, a family of proteins, termed hematopoietic growth factors (HGFs), ${ }^{1}$ stimulates the division and differentiation of committed progenitor cells. This family includes two classes of molecules. The first class stimulates committed progenitor cells restricted to single lineages, and includes macrophage colony stimulating factor (1), granulocyte colony stimulating fac-

Address reprint requests to Dr. Stephen G. Emerson, The University of Michigan Medical Center, MSRB I, Room 5510B, 1150 West Medical Center Drive, Ann Arbor, MI 48109.

Received for publication 22 September 1987 and in revised form 18 March 1988.

1. Abbreviations used in this paper: $\mathrm{B} 1 \mathrm{CM}$, bladder carcinoma 5637 conditional medium; BFU-E, erythroid burst-forming unit; CFUGEMM, granulocyte-erythroid-macrophage-megakaryocyte colony forming unit; CFU-GM, granulocyte-macrophage colony forming unit; CFU-Mk, megakaryocyte colony forming unit; Epo, erythropoietin; GM-CSF, human recombinant granulocyte-macrophage colony stimulating factor; HGF, hematopoietic growth factor; IMDM, Iscove's modified Dulbecco's medium; MoCM, Mo cell line conditional medium.

J. Clin. Invest.

(c) The American Society for Clinical Investigation, Inc.

$0021-9738 / 88 / 10 / 1282 / 06 \quad \$ 2.00$

Volume 82, October 1988, 1282-1287 tor (2), erythropoietin (Epo) (3), and perhaps thrombopoietin (4). The second class of HGFs affects progenitors of several lineages as well as more primitive multipotential progenitor cells.

The first multipotent HGF isolated was granulocyte-macrophage colony stimulating factor (GM-CSF) (5). Picomolar quantitives of GM-CSF provide colony stimulating activities to granulocyte progenitors, macrophage progenitors, bipotent granulocyte-macrophage progenitors, erythroid burst forming units (BFU-E), and multipotential myeloid progenitors. GMCSF is active on progenitor cells from bone marrow, peripheral blood, and fetal liver (6-8).

Recently a cDNA has been cloned from the Gibbon leukemic cell line MLA-144 which encodes a second multipotent HGF, IL-3 (9). The human genome contains a homologous gene, whose predicted protein product differs from the gibbon IL-3 protein by only nine amino acids. The gibbon protein is active on human bone marrow progenitor cells (9), while the activities of purified human IL-3 have yet to be defined.

In this study we examined the effects of human recombinant proteins IL-3 and GM-CSF on highly enriched human progenitor cells. We show that while both hormones have multilineage stimulating activities, their target cell specificities are distinguishable. IL-3 supports the differentiation of a larger number of erythroid and megakarocytic progenitor cells than does GM-CSF, while GM-CSF supports more myeloid progenitors. IL-3 promotes BFU-E division, while GM-CSF appears to support BFU-E survival with little proliferation. In addition, IL-3 promotes the formation of greater numbers of multipotential granulocyte-erythroid-macrophage-megakaryocyte colony forming unit (CFU-GEMM)-derived colonies than does GM-CSF. These data support a model of differentiation in which the acquisition and loss of growth factor responsiveness and lineage specific restriction are parallel but asynchronous programs. In addition these data suggest a potential role for combined GM-CSF/IL-3 therapy in selected clinical settings.

\section{Methods}

Bone marrow samples. Normal human bone marrow was obtained following informed consent by aspiration into preservative-free heparin from adult volunteers, under a protocol approved by the University of Michigan Human Investigation Committee.

Cell separation procedures. The marrow suspensions were centrifuged over Ficoll-Hypaque $\left(1.077 \mathrm{~g} / \mathrm{cm}^{3}\right.$ ) (Pharmacia Fine Chemicals, Piscataway, $\mathrm{NJ}$ ) at $400 \mathrm{~g}$ for $40 \mathrm{~min}$ at $20^{\circ} \mathrm{C}$, the interface mononuclear cells collected, washed three times, and resuspended in Iscove's modified Dulbecco's medium (IMDM) containing 20\% FCS. The cells were incubated in 100-mm tissue culture dishes (Lux, Miles Laboratories, Elkhart, IN) overnight at $37^{\circ} \mathrm{C}$ and the nonadherent cells removed with two gentle washes and centrifuged.

Progenitor cell enrichment by panning. Nonadherent cells were incubated with saturating quantities of a panel of eight murine mono- 
clonal antibodies for $1 \mathrm{~h}$ at $4^{\circ} \mathrm{C}$. The antibodies, their sources, specificities, and the quantities employed per $10^{6}$ total nucleated cells were as follows: anti-Leu-1 (Becton-Dickinson \& Co., Oxnard, CA), T cells, $100 \mathrm{ng}$; anti-Leu-5b (Becton-Dickinson \& Co.), T cells and NK cells, $40 \mathrm{ng}$; anti-Leu-10 (Becton-Dickinson \& Co.), HLA-DQ, $400 \mathrm{ng}$; antiLeu-M1 (Becton-Dickinson \& Co.), mature and precursor myeloid and monocytic cells, $200 \mathrm{ng}$; anti-Leu-12 (Becton-Dickinson \& Co.), B cells, $200 \mathrm{ng}$; anti-CALLA (Becton-Dickinson \& Co.), pre-B cells, 300 ng; TG-1 (courtesy of Peter Beverley [10]), granulocytes, myeloid precursors, some monocytes, $5 \mu \mathrm{l}$ hybridoma supernatant; 4F7 (Laurence Livermore Laboratories, Livermore, CA) glycophorin A, $1 \mu$ l hybridoma supernatant. In some experiments $200 \mathrm{ng}$ of Leu- 18 (anti My-11) (Becton-Dickinson \& Co.), which detects granulocyte-macrophage colony forming units (CFU-GM) and myeloid precursors, was also included. Antibody-coated cells were washed three times to remove excess antibody before panning. Separation of antibody-negative cells (panning) was performed as previously described (6). Anti-Ig plates were prepared by incubating $100 \times 15$-mm plastic culture plates (Lux) with $5 \mathrm{ml}$ affinity purified rabbit anti-mouse $\mathrm{Ig}(100 \mu \mathrm{g} / \mathrm{ml})$ in PBS overnight at $4^{\circ} \mathrm{C}$ and washing with cold PBS before use. Coating the plates with $<250 \mu \mathrm{g}$ antibody resulted in suboptimal cell binding. Murine antibody-labeled, washed cells were suspended in $5 \mathrm{ml}$ PBS at $1-5 \times 10^{7}$ cells $/ \mathrm{ml}$ with $5 \%$ heat-inactivated FCS and incubated over the rabbit anti-mouse Ig coated plates for $1 \mathrm{~h}$ at $4^{\circ} \mathrm{C}$, after which time the nonadherent, antibody negative cells were recovered by gently pipetting without disrupting the antibody-coated cells bound to the plates. Incubation times of $<\mathbf{4 0}$ min were ineffective at allowing maximal cell binding to the plates, while cells could be allowed to adhere to the plates for at least $2 \mathrm{~h}$ without decreased binding or subsequent plating efficiency. Panning removed $90-95 \%$ of bone marrow cells but removed only $0-15 \%$ of the progenitor cells. Progenitor cells were thereby concentrated 9-20-fold by the panning procedure.

Culture conditions. For most experiments, the bone marrow cells were cultured in $0.9 \%$ methylcellulose in IMDM containing $30 \%$ FCS (HyClone Laboratories, Logan, UT), 1\% BSA (Sigma Chemical Co., St. Louis, MO), $10^{-4} \mathrm{M} \beta$-mercaptoethanol. The lot of FCS used was chosen by comparison among six lots: the lot that supported maximal colony growth in the presence of Epo + Mo cell line conditional medium (MoCM), but none without Epo + MoCM, was selected. GM-CSF, IL-3, or MoCM was added to the cultures at their initiation (day 0 ), and erythropoietin was added on day 3. For liquid culture experiments, bone marrow cells were initially incubated in IMDM plus $20 \%$ FCS, with or without GM-CSF or IL-3, for 1-7 d before plating in methylcellulose. Methylcellulose cultures were routinely established at 5,000-10,000 cells/ml in $0.33-\mathrm{ml}$ triplicates in 24-well tissue culture plates (Costar, Cambridge, MA) and incubated at $37^{\circ} \mathrm{C}$ in a high humidity, $5 \% \mathrm{CO}_{2}$ incubator.

Megakaryocyte cultures were performed as described for murine cells $(11,12)$, except that $30 \%$ human plasma replaced the FCS. Briefly, cells were cultured in supplemented McCoy's 5A media containing $30 \%$ heparinized human plasma and $0.25 \%$ bactoagar (Difco, Detroit, MI). Bone marrow cells were cocultivated with conditioned medium from a human bladder carcinoma cell line (5637), IL-3, or GM-CSF. Incubation conditions were identical to those for methylcellulose cultures. Megakaryocyte colonies were defined as greater than three cells/ colony and were identified by their morphological characteristics as described elsewhere (13-15).

BFU-E- and CFU-GM-derived colonies were counted on day 14 , and megakaryocyte colony forming unit (CFU-Mk)- and CFUGEMM-derived colonies were counted on day 17. CFU-GEMM-derived colonies were clearly distinguished from overlapping erythroid and myeloid colonies by tracking the development of individual colonies over the 17-d culture. In addition, the frequency of CFUGEMM-derived colonies was unchanged in cell cultures plated over a range from 1,000 to 20,000 cells per ml. For one series of experiments total colonies and cell numbers per colony were counted on day 3 , and the numbers of subcolonies per BFU-E-derived colonies were counted on day 14 .
Hematopoietic growth factors. Purified recombinant erythropoietin (AmGen, Thousand Oaks, CA) was employed at $2 \mathrm{U}$ per ml. Purified recombinant human GM-CSF (5) was generously provided by the Genetics Institute Pilot Development Laboratory, and purified recombinant human IL-3 (9) was the generous gift of Dr. K. Turner and Dr. R. Kaufman, Genetics Institute, Cambridge, MA. Both of these hematopoietins were produced from engineered Chinese hamster ovary cells and had specific activities of $2-4 \times 10^{6} \mathrm{CML} \mathrm{U} / \mathrm{mg}$ (9). MoCM was prepared from the Mo T lymphoblast cell line (16). Human bladder carcinoma (5637) conditioned media (B1CM) (17) was obtained from carcinoma cells grown in RPMI 1640 containing $0.75 \%$ (wt/vol) glutamine $(100 \times$, Gibco Laboratories, Grand Island, NY) and $2 \%$ FCS. Bladder carcinoma cells were cultured in $75-\mathrm{cm}^{2}$ flasks on Cytodex-3 beads (Pharmacia Fine Chemicals), grown to confluence (on beads), and media conditioned for 3-4 d. After centrifugation, the B1CM was concentrated fivefold by ultrafiltration (YM-10 membrane, Amicon Corp., Danvers, MA), filter sterilized, and stored at $-80^{\circ} \mathrm{C}$.

\section{Results}

Responsiveness of human hematopoietic progenitor cells to $I L-3$. To accurately assess the effects of recombinant human IL-3, we measured its activity on bone marrow cells that had been physically and immunologically depleted of all known accessory cells producing HGFs (6). This adherence and panning procedure, which concentrates progenitor cells 10-30fold, yields target cells that are totally dependent on added HGFs for their survival, division, and differentiation. The addition of increasing concentrations of IL-3 to methylcellulose cultures containing these enriched progenitor cells resulted in a dose-dependent induction of erythroid, myeloid, and mixed erythroid-myeloid colonies. For each lineage, maximal progenitor cell induction was seen at IL-3 concentrations of 2 $\mathrm{ng} / \mathrm{ml}$ or higher (Fig. 1). This concentration was therefore used in all subsequent experiments in assaying IL-3 effects.

Differential responsiveness of human progenitor cells to $I L-3$ and GM-CSF. When assayed using enriched bone marrow progenitor cells, both recombinant human GM-CSF and IL-3 supported erythroid, myeloid, and mixed erythroid-myeloid colonies. GM-CSF supported larger numbers of myeloid colonies than did IL-3, though not as many as with $10 \%$ MoCM (Table I). Both HGFs stimulate granulocyte, macro-

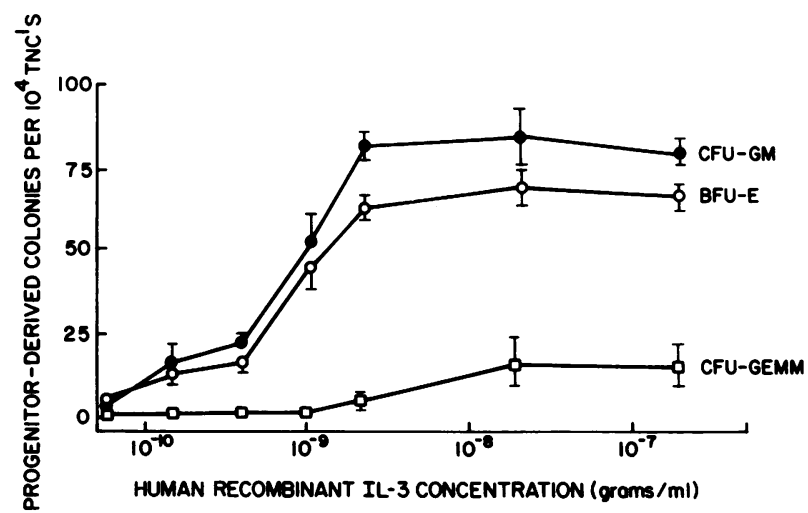

Figure 1. Responsiveness of hematopoietic progenitor cells to IL-3. Human bone marrow progenitor cells were enriched by panning as described in Methods, and cultured in methylcellulose with Epo added on day 3. BFU-E and CFU-GM-derived colonies were enumerated on day 14, and CFU-GEMM-derived colonies on day 17. The data are means \pm SD from one of three similar experiments. 
Table I. Progenitor-derived Hematopoietic Colonies Supported by IL-3 and GM-CSF

\begin{tabular}{lclc}
\hline Growth factor or supernatant & BFU-E* & \multicolumn{1}{c}{ CFU-GM* } & CFU-GEMM* \\
\hline IL-3 $(2 \mathrm{ng} / \mathrm{ml})$ & $1,250 \pm 250$ & $3,100 \pm 520$ & $250 \pm 65$ \\
GM-CSF $(100 \mathrm{ng} / \mathrm{ml})$ & $300 \pm 75$ & $4,200 \pm 850$ & $150 \pm 50$ \\
MoCM $(10 \%)$ & $600 \pm 123$ & $6,000 \pm 1,040$ & $200 \pm 35$ \\
IL-3 + GM-CSF & $1,200 \pm 325$ & $6,200 \pm 940$ & $250 \pm 80$ \\
\hline
\end{tabular}

* Progenitor-derived colonies per $10^{5}$ total nucleated cells cultured. The data presented are means \pm SD from one of five similar experiments.

phage, and mixed granulocyte-macrophage colony formation. The cocultivation of GM-CSF and IL-3 gave no more myeloid colonies than did GM-CSF alone.

In contrast to GM-CSF, IL-3 has a more pronounced effect on early erythroid progenitor cells. In each of six experiments, IL-3 (2 ng/ml) consistently yielded $50-400 \%$ more erythroid bursts than did cultures containing as much as $300 \mathrm{ng} / \mathrm{ml}$ GM-CSF. Additionally, IL-3 supports 20-50\% more BFU-Ederived colonies than does $10 \% \mathrm{MoCM}$. As with granulocyte progenitor cells, the combination of GM-CSF and IL-3 was not additive for erythroid colony formation. Cultures containing IL-3 always yielded 20-100\% more CFU-GEMM-derived colonies than did cultures containing GM-CSF.

IL-3 induces the division and migration of primitive $B F U-E$. The erythroid colonies induced by IL-3 appeared to be somewhat larger and more multicentric than GM-CSF- or MoCM-induced colonies. To further explore this difference, individual erythroid colonies induced by GM-CSF or IL-3 were examined under a magnification of 300 using Hoffman contrast optics, and the number of subcolonies constituting each burst was measured. In cultures containing GM-CSF, bursts ranged in composition from single unicentric colonies to multicentric colonies with 35 subcolonies. Most of the colonies contained subcolonies, with mean $=10.4$ subcolonies and median $=9$ subcolonies. The range of erythroid bursts induced by IL-3 also included small unicentric colonies, but included larger and more numerous multicentric colonies than did cultures containing GM-CSF (mean = 15.4 subcolonies, median = 14) (Fig. $2 \mathrm{~A}$ ).

To focus on the proliferative effects of IL-3 and GM-CSF on the earliest progeny of erythroid progenitor cells, BFU-Ederived clusters were examined after $3 \mathrm{~d}$ of culture. BFU-E were enriched using an antibody cocktail containing anti-Leu 18 (18). This antibody bound to most CFU-GM, CFU-G, and CFU-M as well as myeloid precursors, so that the great majority of colonies that developed were erythroid. Enriched progenitor cells were plated in methylcellulose in the presence of IL-3 or GM-CSF. Individual clusters of cells were identified and the numbers of cells per cluster enumerated on day 3 of the cultures. Clusters induced by GM-CSF were generally small, containing two to four cells. IL-3 induced not only similar small clusters, but also larger clusters containing six or more cells (Fig. 2 B). These data suggested that IL-3 exerted a stronger proliferative effect on BFU-E than did GM-CSF.

The effects of IL-3 and GM-CSF on BFU-E division were directly compared by incubation of these factors in liquid cultures with enriched bone marrow progenitor cells before
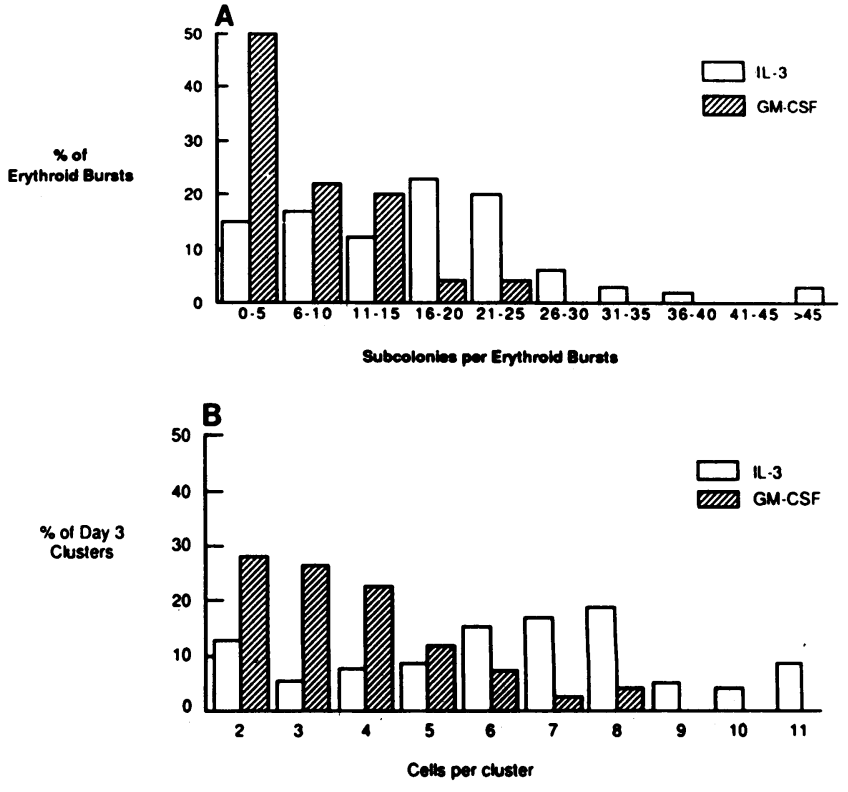

Figure 2. IL-3 promotes BFU-E division and migration. (A) Subcolonies per colony. Mature (d14) erythroid colonies were examined under $300 \times$ Hoffman contrast optics, and the numbers of subcolonies per colony were enumerated. 123 total GM-CSF-supported erythroid colonies and 144 IL-3-supported colonies were examined. $(B)$ Day 3 cluster size. Erythroid progenitor cells were specifically enriched by adding Leu 18 to the monoclonal antibody depletion cocktail, and the number of undifferentiated blast cells per cell cluster enumerated after $3 \mathrm{~d}$ of methylcellulose culture. 108 total GMCSF-supported and $125 \mathrm{IL}$-3-supported clusters were analyzed, of which $96 \%$ subsequently were found to be erythroid bursts, $1 \%$ myeloid colonies, and 3\% multilineage (CFU-GEMM-derived) colonies.

short-term colony assays. Under these conditions, the induction of BFU-E self-renewal or commitment was assayed by the appearance of more BFU-E-derived colonies in the subsequent cultures. Incubation of enriched progenitor cells in the presence of GM-CSF sustained their survival for 72-96 h, allowing similar numbers of bursts to develop as observed in cultures that were initiated before liquid culture. However, there was no increase in BFU-E by preincubation with GMCSF. In contrast, preincubation in IL-3 led to increasing numbers of bursts in the subsequent semisolid colony assays. Liquid culture without GM-CSF or IL-3 led to the loss of all subsequently clonable BFU-E (Fig. 3). This greater effect of IL-3 versus GM-CSF on BFU-E proliferation in suspension was observed over a wide range of concentrations of both recombinant hormones (Fig. 4), and was seen throughout the time course of the culture at all concentrations. Thus, while GM-CSF appeared to maintain numbers of BFU-E without inducing division, IL-3 increased BFU-E either by actively inducing self-renewal and expansion or by inducing differentiation from multilineage progenitors to BFU-E in liquid culture.

Stimulation of megakaryocyte colony formation with recombinant HGFs. Both IL-3 and GM-CSF supported the development of megakaryocyte colonies. Colony development was limited with colonies being comprised of 3-50 relatively immature cells. In each experiment IL-3 supported higher numbers of megakaryocyte colonies than did GM-CSF (Table 


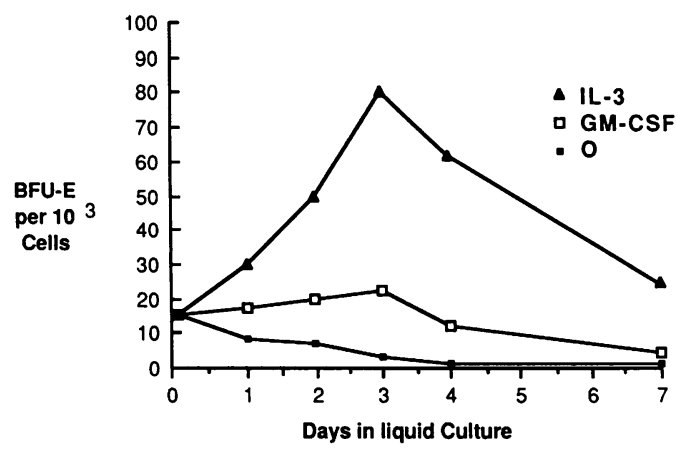

Figure 3. IL-3 promotes BFU-E pool expansion in liquid culture: kinetics. Enriched bone marrow progenitor cells were cultured in liquid suspension in the presence of $1 \mathrm{ng} / \mathrm{ml} \mathrm{IL-3,100} \mathrm{ng/ml} \mathrm{GM-CSF,}$ or no added HGF for up to $7 \mathrm{~d}$ in liquid culture. Aliquots were removed, placed into methylcellulose cultures for $14 \mathrm{~d}$, and erythroid bursts enumerated. The data shown display the number of erythroid bursts per $10^{3}$ cells originally placed into liquid culture, and are from one of three similar experiments.

II). Extended dose response experiments confirmed that this increased megakaryocytopoietic effect of IL-3 was not due to increased factor avidity alone, but also to a larger number of susceptible CFU-Mk. The megakaryocytopoietic effect of GM-CSF was maximal at $100-300 \mathrm{ng} / \mathrm{ml}$, and was actually substantially lower at $500 \mathrm{ng} / \mathrm{ml}$ (Fig. 5). No additive or synergistic effects between these two factors were observed in five experiments over ranges of GM-CSF concentrations from 1 to $300 \mathrm{mg} / \mathrm{ml}$ and IL-3 concentration from 0.1 to $10 \mathrm{ng} / \mathrm{ml}$. Maximal megakaryocyte colony formation was always seen with IL-3, and the simultaneous inclusion of GM-CSF and IL-3 never stimulated the proliferation of more megakaryocytic colonies than did the same concentration of IL-3 alone (Fig. 6).

\section{Discussion}

Both purified GM-CSF and IL-3 have distinct multilineage colony stimulating activities, supporting the survival and proliferation of primitive BFU-E, CFU-GM, CFU-Mk, and multipotential CFU-GEMM. While GM-CSF supports greater

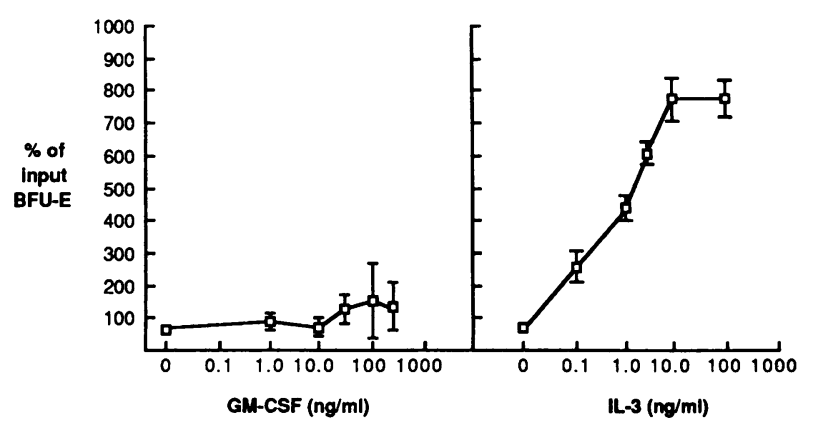

Figure 4. IL-3 promotes BFU-E pool expansion in liquid culture: dose response. Enriched bone marrow progenitors were cultured in suspension for $3 \mathrm{~d}$ before methylcellulose cultures, and BFU-E were enumerated $14 \mathrm{~d}$ later, as in Fig. 3. The data shown are means \pm SD of the number of erythroid bursts as a percentage of control cultures plated in methylcellulose before suspension, and are from one of four similar experiments.
Table II. Human Megakaryocyte Progenitor Cell Responsiveness to IL-3

\begin{tabular}{|c|c|c|c|c|c|c|c|c|}
\hline \multirow{3}{*}{$\begin{array}{l}\text { Growth factor or } \\
\text { supernatant }\end{array}$} & \multicolumn{8}{|c|}{ Donor (Exp. No.) } \\
\hline & \multicolumn{4}{|c|}{ CFU-Mk } & \multicolumn{4}{|c|}{ CFU-GM } \\
\hline & 1. & 2. & 3. & $\overline{\mathbf{X}} \pm \mathrm{SD}$ & 1. & 2. & 3. & $\overline{\mathrm{X}} \pm \mathrm{SD}$ \\
\hline \multirow{2}{*}{\multicolumn{9}{|c|}{ 2. IL-3 }} \\
\hline & & & & & & & & \\
\hline $100 \mathrm{pg} / \mathrm{ml}$ & 0 & 3 & 0 & $1 \pm 2$ & 93 & 29 & 72 & $65 \pm 32$ \\
\hline $1,000 \mathrm{pg} / \mathrm{ml}$ & 21 & 8 & 0 & $10 \pm 11$ & 83 & 61 & 66 & $70 \pm 11$ \\
\hline $2,000 \mathrm{pg} / \mathrm{ml}$ & 35 & 37 & 11 & $28 \pm 15$ & 43 & 75 & 35 & $51 \pm 21$ \\
\hline 3. $\mathrm{GM}-\mathrm{CSF}(300 \mathrm{ng} / \mathrm{ml})$ & 20 & 3 & 5 & $9 \pm 9$ & 100 & 53 & 72 & $75 \pm 23$ \\
\hline
\end{tabular}

* CFU-Mk- and CFU-GM-derived colonies per $10^{5}$ total nucleated cells cultured. Megakaryocyte progenitor cells were cultured as in Methods. Results for each donor are means of three to five replicate cultures.

numbers of myeloid colonies than does IL-3, IL-3 has a stronger proliferative effect on BFU-E and CFU-Mk, as well as multilineage CFU-GEMM. The target cells for these studies were enriched progenitor cells from which all known accessing cells had been depleted. Cultures failed to generate colonies when no added growth factor was added, suggesting that no such activities were present in the cultures. Nevertheless, it is indeed possible that some of the effects of GM-CSF and IL-3 were indirect effects mediated through intermediary cells or in concert with costimulating hormones.

The greater burst-promoting activity of IL-3 is apparent both in the number of bursts generated and in the size and composition of the bursts. IL-3 stimulated the generation of larger multicentric bursts than observed with GM-CSF. Close examination of erythroid colony formation during the first $3 \mathrm{~d}$ demonstrated that IL-3 exerted a stronger early proliferative effect on BFU-E than does GM-CSF.

The greater proliferative effect of IL-3 was further demonstrated in the liquid culture incubations. During $3 \mathrm{~d}$ of liquid culture, IL-3 increased the frequency of BFU-E, while GMCSF merely sustained input numbers of these cells. These data suggest that IL-3 either stimulates self-renewal of BFU-E or, alternatively, drives erythroid commitment from antecedent

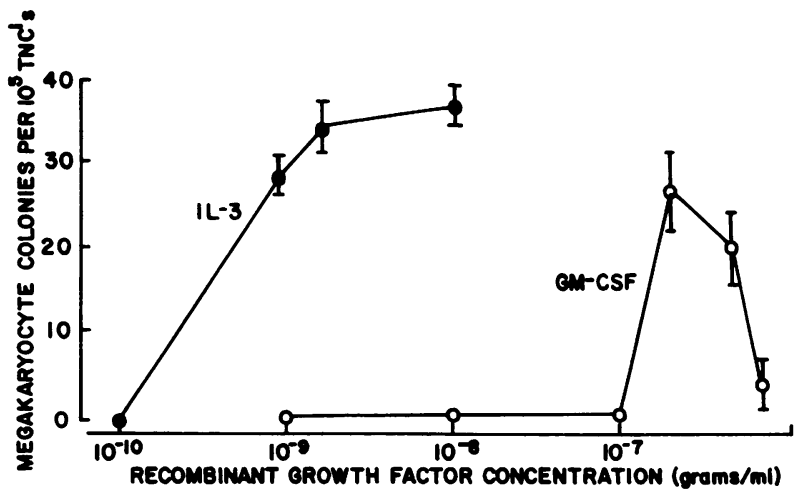

Figure 5. Comparative responsiveness of megakaryocyte progenitor cells to IL-3 and GM-CSF. Progenitor cells cultured as in Methods. Results are mean $\pm S D$ of three to five replicate cultures (per concentration) of parallel cell cultures on a single donor, and are taken from one of five similar experiments. 


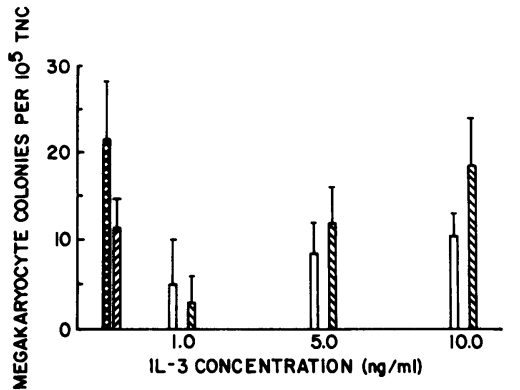

Figure 6. Interaction between IL-3 and GMCSF in megakaryocyte colony formation. Human enriched bone marrow progenitor cells were cultured in the presence of varying concentrations of IL-3 $(1.0-10.0 \mathrm{ng} / \mathrm{ml})$ and GM-CSF $(200 \mathrm{ng} / \mathrm{ml}$ [open bars] or 300 $\mathrm{ng} / \mathrm{ml}$ [left diagonal hatched bars]). The bars to the left of the graph are the data for optimal concentrations of IL-3 only $(1.0 \mathrm{ng} / \mathrm{ml}$ [cross-hatched bar]) and GM-CSF only $(300 \mathrm{ng} / \mathrm{ml}$ [right diagonal hatched bar]) in this experiment. The data shown are from triplicate cultures and are taken from one of five similar experiments.

plurimultipotent cells (CFU-GEMM). In contrast, the ability of GM-CSF to stimulate BFU-E self-renewal is more limited, rather supporting the survival of BFU-E until they become erythropoietin responsive.

Our observations on the direct proliferative effects of IL-3 and the ability of GM-CSF to support the survival of primitive BFU-E extend and confirm similar results by Metcalf et al. using the murine system, which showed that partially purified murine GM-CSF and crude spleen cell-conditioned medium synergized to support BFU-E differentiation $(19,20)$. In particular, partially purified GM-CSF appeared to support the survival of clonable BFU-E for $48 \mathrm{~h}$, at which time spleen cell-conditioned medium addition stimulated the subsequent development of erythroid bursts (20). Recent work with purified recombinant human GM-CSF and Epo has confirmed that this synergism actually requires GM-CSF doses one-tenth as great as for myelopoietic effects and is directly attributable to $\operatorname{GM}-\operatorname{CSF}(6,7)$.

Both IL-3 and GM-CSF are capable of stimulating megakaryocyte colony formation. As with the erythroid (BFU-E) and multipotential (CFU-GEMM) progenitor cells, greater numbers of megakaryocyte progenitor cells appear to be responsive to IL-3 than to GM-CSF. This observation implies that either some IL-3-responsive CFU-Mk are not GM-CSF responsive or, alternatively, distinct subpopulations of cells respond to each HGF. While the data do not allow clear distinction between these alternatives, the lack of an additive effect between IL-3 and GM-CSF coincubation argues in favor of a subpopulation of IL-3-responsive CFU-Mk being GMCSF responsive. In contrast to these observations, Robinson et al. have shown an additive effect with murine IL-3 and GMCSF on megakaryocyte colony development (21). Such differences may be species specific.

One interesting conclusion from our data is that a progenitor cell's susceptibility to growth factor responsiveness is not a strict property of restricted lineage potential. Some, but not all, restricted erythroid or megakaryocytic progenitor cells are supported by GM-CSF, as are some but not all multipotential CFU-GEMM. Lineage restriction and growth factor responsiveness are acquired more or less in parallel, and not by strictly sequenced or synchronized developmental switches.

From a clinical perspective, these data suggest that GMCSF and IL-3 might be expected to have overlapping in vivo activities. While each hormone would have effects on all my- eloid lineages, GM-CSF would be expected to have a stronger neutrophilic effect whereas IL-3 might have a stronger thrombopoietic effect. In clinical scenarios such as bone marrow transplantation and chemotherapy-induced nadirs, combined therapy with GM-CSF and IL-3 might be required for optimal amelioration of neutrophil and platelet deficiency.

In summary, these data indicate that human recombinant GM-CSF and IL-3 have overlapping but distinct spectra of activities. These data further suggest that hematopoietic differentiation progresses through two parallel and loosely synchronized programs: the acquisition and loss of HGF responsiveness, and restriction to lineage commitment. Future clinical applications might take advantage of distinct activities to achieve optimal benefits by combined treatment with both hormones.

\section{References}

1. Wong, G. G., P. A. Temple, A. C. Leary, J. S. Witek-Giannotti, Y. C. Yang, A. B. Ciarletta, M. Chung, P. Murtha, R. Kriz, R. J. Kaufman, C. R. Ferenz, B. S. Sibley, K. J. Turner, R. M. Hewick, S. C. Clark, N. Yanai, H. Yokota, M. Yamada, M. Saito, K. Motoyoshi, and F. Takaku. 1987. Human CSF-1: molecular cloning and expression of 4-kb cDNA encoding the human urinary protein. Science (Wash. DC). 235:1504-1508.

2. Welte, K., E. Platzer, L. Lu, J. L. Gabrilove, E. Levi, R. Mertelsmann, and M. A. S. Moore. 1985. Purification and biochemical characterization of human pluripotent hematopoietic colony-stimulating factor. Proc. Natl. Acad. Sci. USA. 82:1526-1530.

3. Jacobs, K., C. Shoemaker, R. Rudersdorf, S. D. Neill, R. J. Kaufman, A. Mufson, J. Seehra, S. S. Jones, R. Hewick, E. F. Fritsch, M. Kawakita, T. Shimizu, and T. Miyake. 1985. Isolation and characterization of genomic and cDNA clones of human erythropoietin. Nature (Lond.). 313:806-810.

4. Tayrien, G., and R. D. Rosenberg. 1987. Purification and properties of a megakaryocyte stimulatory factor present both in the serum-free conditioned medium of human embryonic kidney cells and in thrombocytopenic plasma. J. Biol. Chem. 262:3262-3268.

5. Wong, G. G., J. S. Witek, P. A. Temple, K. M. Wilkens, A. C. Leary, D. P. Luxenberg, S. S. Jones, E. L. Brown, R. M. Kay, E. D. Orr, C. Shoemaker, D. W. Golde, R. J. Kaufman, R. M. Hewick, E. A. Wang, and S. C. Clark. 1985. Human GM-CSF: molecular cloning of the complementary DNA and purification of the natural and recombinant proteins. Science (Wash. DC). 228:81-815.

6. Emerson, S. G., C. A. Sieff, E. A. Wang, G. G. Wong, S. C. Clark, and D. G. Nathan. 1985. Purification of fetal hematopoietic progenitors and demonstration of recombinant multipotential colony-stimulating activity. J. Clin. Invest. 76:1286-1290.

7. Donahue, R. E., S. G. Emerson, E. A. Wang, G. G. Wong, S. C. Clark, and D. G. Nathan. 1985. Demonstration of burst promoting activity of recombinant human GM-CSF on circulating erythroid progenitors using an assay involving the delayed addition of erythropoietin. Blood. 66:1479-1482.

8. Sieff, C. A., S. G. Emerson, R. E. Donahue, D. G. Nathan, E. A. Wang, G. G. Wong, and S. C. Clark. 1985. Human recombinant GM-CSF is a multilineage hemopoietin. Science (Wash. DC). 230:1171-1173.

9. Yang, Y. C., A. B. Ciarletta, P. A. Temple, M. P. Chung, S. Kovacic, J. S. Witek-Gianotti, A. C. Leary, R. Kriz, R. E. Donahue, G. G. Wong, and S. C. Clark. 1986. Human IL-3 (multi-CSF): identification by expression cloning of a novel hematopoietic growth factor related to murine IL-3. Cell. 47:3-10.

10. Beverley, P. C. L., D. Linch, and D. Delia. 1980. Isolation of human haematopoietic progenitor cells using monoclonal antibodies. Nature (Lond.). 287:332-333.

11. Long, M. W., L. L. Gragowski, C. H. Heffner, and L. A. Boxer. 
1985. Phorbol diesters stimulate the development of an early murine progenitor cell. J. Clin. Invest. 76:431-438.

12. Long, M. W., J. E. Smolen, P. Szczepanski, and L. A. Boxer. 1984. Role of phorbol diesters in in vitro murine megakaryocyte colony formation. J. Clin. Invest. 74:1686-1692.

13. Messner, H. A., N. Jamal, and C. Izaguirre. 1982. The growth of large megakaryocyte colonies from human bone marrow. J. Cell. Physiol. 1:45-51.

14. Kimura, H., S. A. Burstein, J. S. Thorning, L. A. Powell, L. A. Harker, P. J. Fialkow, and J. W. Adamson. 1984. Human megakaryocytic progenitors (CFU-M) assayed in methylcellulose: physical characteristics and requirements for growth. J. Cell. Physiol. 118:87-96.

15. Solberg, L. A., Jr., N. Jamal, and J. A. Messner. 1985. Characterization of human megakaryocytic formation in human plasma. $J$. Cell. Physiol. 124:66-74.

16. Golde, D. W., N. Bersch, S. G. Quan, and A. J. Lusis. 1980. Production of erythroid potentiating activity by a human T-lymphoblast cell line. Proc. Natl. Acad. Sci. USA. 77:593-596.

17. Myers, C. D., F. E. Katz, G. Joshi, and J. L. Millar. 1984. A cell line secreting stimulating factors for CFU-GEMM culture. Blood. 64:152-155.

18. Strauss, L. C., C. Brovall, M. J. Fackler, J. F. Schwartz, J. H. Shaper, M. R. Loken, and C. I. Civin. 1986. Antigenic analysis of hematopoiesis. IV. The My-11 hematopoietic cell surface antigen is expressed by myelomonocytic and lymphoid, but not erythroid progenitor cells. Exp. Hematol. (NY). 14:935-945.

19. Metcalf, D. E., G. R. Johnson, and A. W. Burgess. 1980. Direct stimulation by purified GM-CSF of the proliferation of multipotential and erythroid precursor cells. Blood. 55:138.

20. Metcalf, D. E., and G. R. Johnson. 1979. Interactions between purified GM-CSF, purified erythropoietin, and spleen conditioned medium on hematopoietic colony formation in vitro. J. Cell. Physiol. 99:159-174.

21. Robinson, B. E., H. E. McGrath, and P. J. Quesenberry. 1987. Recombinant murine granulocyte macrophage colony-stimulating factor has megakaryocyte colony-stimulating activity and augments megakaryocyte colony stimulation by interleukin 3. J. Clin. Invest. 79:1648-1652. 